\title{
Dietary intake of $\alpha$-linolenic acid and low ratio of $n-6: n-3$ PUFA are associated with decreased exhaled NO and improved asthma control
}

\author{
Renata Barros $^{1 *}$, André Moreira ${ }^{2,3}$, João Fonseca ${ }^{2,4}$, Luís Delgado ${ }^{2,3}$, M. Graça Castel-Branco ${ }^{2}$, \\ Tari Haahtela ${ }^{5}$, Carla Lopes ${ }^{6}$ and Pedro Moreira ${ }^{1,7}$ \\ ${ }^{1}$ Faculty of Nutrition and Food Sciences, University of Porto, Rua Dr. Roberto Frias, 4200-465 Porto, Portugal \\ ${ }^{2}$ Department of Immunoallergology, Hospital S. João, Porto, Portugal \\ ${ }^{3}$ Department of Immunology, Faculty of Medicine, University of Porto, Porto, Portugal \\ ${ }^{4}$ Department of Biostatistics and Medical Informatics, Faculty of Medicine, University of Porto, Porto, Portugal \\ ${ }^{5}$ Skin and Allergy Hospital, Helsinki University Central Hospital, Helsinki, Finland \\ ${ }^{6}$ Department of Hygiene and Epidemiology, University of Porto Medical School, Porto, Portugal \\ ${ }^{7}$ Research Centre in Physical Activity and Leisure - CIAFEL, University of Porto, Porto, Portugal
}

(Received 16 August 2010 - Revised 13 January 2011 - Accepted 17 January 2011 - First published online 29 March 2011)

\section{Abstract}

As recently described, adherence to the Mediterranean diet is associated with improved asthma control. However, evidence of how specific nutrients such as fatty acids and antioxidants may affect this relationship remains largely unknown. We aimed to examine the association between dietary intake of fatty acids and antioxidants and asthma control. A cross-sectional study was developed in 174 asthmatics, mean age of 40 (SD 15) years. Dietary intake was obtained by a FFQ, and nutritional content was calculated using Food Processor Plus ${ }^{\mathrm{TM}}$ software (ESHA Research, Inc., Salem, OR, USA). Good asthma control was defined by the combination of forced expiratory volume during the first second, exhaled NO (eNO) and Asthma Control Questionnaire (ACQ) score (control: forced expiratory volume in the first second $\geq 80 \%$; eNO $\leq 35 \mathrm{ppb}$; ACQ $<1 \cdot 0$, scale $0-6$ score). Multiple linear and logistic regression models were performed to analyse the associations between nutrients and asthma outcomes, adjusting for confounders. A high $n-6: n-3$ PUFA ratio predicted high eNO, whereas high intakes of $n-3$ PUFA, $\alpha$-linolenic acid (ALA) and SFA were associated with low eNO. Odds for controlled asthma improved along with an increased intake of $n$-3 PUFA (OR 0.14, 95\% CI 0.04, 0.45; $P$ for trend=0.001), SFA (OR 0.36, $95 \%$ CI 0.13, 0.97; $P$ for trend=0.047) and ALA (OR 0.18, $95 \%$ CI $0.06,0.58 ; P$ for trend=0.005). A high $n-6: n-3$ PUFA ratio increased the odds for uncontrolled asthma (OR 3.69, 95\% CI 1.37, 9.94; $P$ for trend=0.009), after adjusting for energy intake, sex, age, education and use of inhaled corticosteroids. Higher intakes of $n-3$ PUFA, ALA and SFA were associated with good asthma control, while the risk for uncontrolled asthma increased with a higher $n-6: n-3$ PUFA ratio. The present results introduce a protective effect of ALA in asthma control, independent of marine $n-3$ fatty acids, and provide a rationale to dietary intervention studies in asthma.

\section{Key words: Asthma: Airway inflammation: Diet: Fatty acids: $\alpha$-Linolenic acid: Antioxidants}

The increase in asthma prevalence in the last decades has been suggested to be related to environment and lifestyle changes, from which diet and physical activity (PA) appear as obvious ${ }^{(1)}$. The following two dietary hypotheses have been proposed: (1) an increase in the consumption of vegetable oils and margarines and a decline in fat of animal origin and fish consumption shifting the $n-6: n-3$ ratio of dietary PUFA from $1: 1$ to $15-17: 1^{(2)}$; (2) a decrease in the intake of fresh fruit, vegetables and whole cereals leading to a reduction in dietary antioxidants ${ }^{(3)}$. Several non-experimental studies have provided evidence supporting the lipid ${ }^{(2)}$ and the antioxidant $^{(3)}$ hypotheses; however, the same has not been reported in intervention trials. Potential benefits of marine $n$-3 PUFA, namely EPA $(20: 5 n-3)$ and DHA (22:6n-3), in inflammatory modulation and asthma have been proposed, whereas the link with the precursor $\alpha$-linolenic acid (ALA; $18: 3 n-3)$ is still scarce.

We have recently reported that high adherence to a Mediterranean dietary pattern was associated with improved asthma control $^{(4)}$. This was particularly relevant as asthma control definition incorporated symptoms, lung function and airway inflammation $^{(5)}$. Among Mediterranean diet food items, nuts

Abbreviations: ACQ, Asthma Control Questionnaire; ALA, $\alpha$-linolenic acid; eNO, exhaled NO; FEV1, forced expiratory volume in the first second; PA, physical activity.

*Corresponding author: R. Barros, fax +351 22 5074329, email renatabarros@fcna.up.pt 
(high in ALA) and fresh fruit emerged as positively associated with lung function and asthma control, respectively. However, few data exist on the associations of individual fatty acids, micronutrients and asthma control.

In the present study, we aimed to investigate the association between several types of fatty acids, antioxidant micronutrients and asthma control, measured by symptoms, lung function and airway inflammation, and we hypothesised that $n-3$ PUFA and antioxidant micronutrients provided from the diet could be associated with improved asthma control in asthmatic patients.

\section{Materials and methods}

\section{Participants and study design}

A total of 219 consecutive patients, older than 16 years old, attending an outpatient Asthma and Allergy clinic at a University Hospital, with a medical diagnosis of asthma, were invited to participate in a cross-sectional study. Exclusion criteria were food allergy, changing of dietary patterns in the last 12 months, pregnancy, presence of diseases which involved specific nutritional therapy and dietary planning, acute illness in the last 4 weeks or inability to comply with the measurement instruments. The present study was conducted according to the guidelines laid down in the Declaration of Helsinki, and all procedures involving human patients were approved by the Institutional ethics committee. Written informed consent was obtained from all patients before inclusion.

\section{Nutritional intake}

Dietary intake was obtained by a self-administered, semiquantitative FFQ, validated for Portuguese adults ${ }^{(6)}$. The FFQ is an 86-item questionnaire that assessed usual dietary intake over the previous 12 months, including usual food groups and beverages. Food intake was estimated by multiplying the frequency of consumption (about nine possibilities from 'never or less than 1 time/month', to ' 6 or more times/d') by the weight of the standard portion size of the food item. A seasonal variation factor was considered for foods in which production and consumption are not regular over the year (mean of 3 months). Nutritional intake was calculated using an adapted Portuguese version of the software Food Processor Plus ${ }^{\circledR}$ (ESHA Research, Inc., Salem, OR, USA), nutritional analysis software that converts food intake into total energy and nutrients, based on food composition tables available from the US Department of Agriculture and national data from typical Portuguese foods. Dietary intake of different types of fatty acids ( $n-3$ and $n-6$ PUFA, SFA and MUFA) and micronutrients involved in antioxidant status and potentially relevant for asthma (vitamins E, C, carotene, retinol, $\mathrm{Mg}$ and $\mathrm{Zn}$ ) ${ }^{(1)}$ were selected as primary independent variables of interest. Although Se plays a role as a cofactor of glutathione peroxidase and as the potential suppressor of asthma inflammation, it was not considered in the nutritional analysis. Considering the wide variation in the content of the major Se food sources (depending on the geographic origin and soil levels), food composition data for Se measure by FFQ are considered unreliable ${ }^{(7)}$.

\section{Anthropometry and physical activity assessment}

BMI was calculated after body weight and height measurements with the subject lightly clothed and barefooted, using a mechanical balance with a stadiometer (Seca model $700{ }^{\circledR}$; Seca Headquarter, Hamburg, Germany). Weight and height were determined to the nearest $0.1 \mathrm{~kg}$ and $0.5 \mathrm{~cm}$, respectively. BMI was calculated as the weight $(\mathrm{kg})$ divided by the square of the height $\left(\mathrm{m}^{2}\right)$.

PA was measured using the International Physical Activity Questionnaire - short version ${ }^{(8)}$. The short $7 \mathrm{~d}$ self-administered version is a seven-item questionnaire that provides information about the frequency and duration of four domains: sedentary activity, time spent walking, and moderate- and vigorous-intensity PA. PA within domains was estimated by weighting the reported frequency (events/week) by duration (min/event) and by a metabolic equivalent level assigned to each activity (walking $=3 \cdot 3$; moderate-intensity $\mathrm{PA}=4 \cdot 0$ and vigorous-intensity $\mathrm{PA}=8 \cdot 0$ ). A combined total PA was computed as the sum of the activity domain scores (total PA= walking + moderate-intensity $\mathrm{PA}+$ vigorous-intensity $\mathrm{PA}$ ) and reported as a continuous measure (total PA score $=$ total metabolic equivalent-min/week).

\section{Asthma control and quality of life: definitions and assessment}

Asthma control was defined by combining the results of lung function, exhaled NO (eNO) and the Asthma Control Questionnaire (ACQ) score ${ }^{(5)}$. Subjects were classified as having 'controlled' asthma if simultaneously they had forced expiratory volume in the first second $(\mathrm{FEV} 1) \geq 80 \% \operatorname{predicted}^{(9)}$, eNO $\leq 35 \mathrm{ppb}^{(10)}$ and ACQ score below $1 \cdot 00^{(11)}$. If any of these features were not present, subjects were classified as 'non-controlled'. Lung function was measured by the determination of FEV1 using PIKO- ${ }^{\circledR}$ (Ferraris Respiratory Europe Limited, Hertford, Herts, UK) ${ }^{(12)}$. Patients were asked to perform a set of three technically acceptable manoeuvres, and the highest FEV1 measurement was registered and expressed as percentage predicted, as recommended by the American Thoracic Society.

eNO was measured with the $\mathrm{NIOX}^{\circledR}$ system (Aerocrine, Stockholm, Sweden), using the online technique recommended by the American Thoracic Society ${ }^{(13)}$, at a flow rate of $50 \mathrm{ml} / \mathrm{s}$.

The seven-item ACQ was designed to assess clinical asthma control during the previous week. A seven-point scale $(0=$ no impairment, $6=$ maximum impairment) was used, and the score was calculated as the mean of the seven items, ranging from 0 (totally controlled) to 6 (severely uncontrolled) ${ }^{(14)}$.

Asthma quality of life was measured by the asthma life quality test, developed by the American College of Allergy, Asthma and Immunology, and validated in Portuguese ${ }^{(15)}$. The selfadministered asthma life quality test includes twenty questions of dichotomous answer (yes/no) assessing six domains: activity and sleep; symptoms; triggers; unscheduled health care use; medication; psychological. Total score was calculated as the sum of affirmative responses, ranging from 0 to 20 (lower values indicate better asthma quality of life). 
Table 1. Characteristics of participants according to asthma control ${ }^{*}$

(Mean values, standard deviations, number of subjects and percentages)

\begin{tabular}{|c|c|c|c|c|c|}
\hline & \multicolumn{2}{|c|}{$\begin{array}{l}\text { Controlled asthma } \\
\qquad(n 40)\end{array}$} & \multicolumn{2}{|c|}{$\begin{array}{l}\text { Non-controlled asthma } \\
(\text { (n 134) }\end{array}$} & \multirow[b]{2}{*}{$P$} \\
\hline & Mean & SD & Mean & SD & \\
\hline \multicolumn{6}{|l|}{ Demographic } \\
\hline Age (years) & $42 \cdot 9$ & $13 \cdot 4$ & $39 \cdot 7$ & $15 \cdot 6$ & $0.206 \dagger$ \\
\hline $\operatorname{Sex}(n)$ & & & & & $0.445 \ddagger$ \\
\hline Female & \multicolumn{2}{|c|}{31} & \multicolumn{2}{|c|}{111} & \\
\hline Male & \multicolumn{2}{|c|}{9} & \multicolumn{2}{|c|}{23} & \\
\hline \multicolumn{6}{|l|}{$\leq 4$ years } \\
\hline$n$ & \multicolumn{2}{|c|}{14} & \multicolumn{2}{|c|}{55} & \\
\hline$\%$ & \multicolumn{2}{|c|}{35} & \multicolumn{2}{|c|}{41} & \\
\hline $5-9$ years & & & & & \\
\hline$n$ & & & & & \\
\hline$\%$ & & & & & \\
\hline$\geq 10$ years & & & & & \\
\hline$n$ & & & & & \\
\hline$\%$ & & & & & \\
\hline BMI $\left(\mathrm{kg} / \mathrm{m}^{2}\right)$ & $26 \cdot 8$ & 4.5 & $27 \cdot 3$ & $5 \cdot 3$ & $0.620 \dagger$ \\
\hline Physical activity (MET-min/week) & & & & & $0.328 \|$ \\
\hline Median & & & & & \\
\hline Range & & & & & \\
\hline Present smoker & & & & & $0 \cdot 119 \ddagger$ \\
\hline$n$ & & & & & \\
\hline$\%$ & & & & & \\
\hline Clinical & & & & & \\
\hline Atopic & & & & & $0 \cdot 138 \ddagger$ \\
\hline$n$ & & & & & \\
\hline$\%$ & & & & & \\
\hline Allergic rhinitis & & & & & $0.753 \ddagger$ \\
\hline$n$ & & & & & \\
\hline$\%$ & & & & & \\
\hline Present ICS & & & & & $0.007 \ddagger^{\star *}$ \\
\hline$n$ & & & & & \\
\hline$\%$ & & & & & \\
\hline Exhaled NO (ppb) & & & & & $<0.001 \|^{\star \star}$ \\
\hline Mean & & & & & \\
\hline $95 \% \mathrm{Cl}$ & 16 & & 28. & & \\
\hline FEV1 & $103 \cdot 8$ & $22 \cdot 3$ & 82.7 & $22 \cdot 3$ & $<0.001 \dagger^{\star \star}$ \\
\hline ALQ score & $10 \cdot 1$ & $3 \cdot 8$ & $11 \cdot 7$ & $4 \cdot 2$ & $0.027 \dagger^{\star \star}$ \\
\hline ACQ score & 0.4 & 0.3 & 1.5 & 1.0 & $<0.001 \dagger^{\star \star}$ \\
\hline Nutritional intake & & & & & \\
\hline Total energy intake $(\mathrm{kJ} / \mathrm{d})$ & 14121 & 4091.95 & 13459.92 & 5133.76 & $0.457 \dagger$ \\
\hline Protein (\% TEV) & $16 \cdot 3$ & 2.9 & $15 \cdot 8$ & 3.7 & $0.433 t$ \\
\hline Total carbohydrates (\% TEV) & $35 \cdot 6$ & $6 \cdot 7$ & $35 \cdot 0$ & $7 \cdot 6$ & $0.667 \dagger$ \\
\hline Sugars§ & $15 \cdot 0$ & $5 \cdot 8$ & 14.5 & $5 \cdot 2$ & $0.638 \dagger$ \\
\hline Total fat (\% TEV) & $46 \cdot 2$ & $8 \cdot 7$ & 43.7 & $9 \cdot 6$ & $0.148 \dagger$ \\
\hline PUFA & $6 \cdot 2$ & 1.4 & $6 \cdot 3$ & 1.6 & $0.752 \dagger$ \\
\hline$n-6$ & 4.9 & 1.4 & $5 \cdot 1$ & 1.5 & $0.614 \dagger$ \\
\hline$n-3$ & 0.7 & 0.1 & 0.6 & 0.2 & $0.090 \dagger$ \\
\hline ALA $(g)$ & 2.09 & 0.73 & 1.80 & 0.77 & $0.032 \dagger$ \\
\hline EPA $(g)$ & & & & & $0.016 \|$ \\
\hline Median & & & & & \\
\hline Range & 0.0 & & 0.0 & & \\
\hline DHA (g) & & & & & $0.021 \|$ \\
\hline Median & & & & & \\
\hline Range & 0.1 & & 3.9 & & \\
\hline$E P A+D H A(g)$ & & & & & $0.020 \|$ \\
\hline Median & & & & & \\
\hline Range & 1.4 & & & & \\
\hline MUFA & $16 \cdot 7$ & 3.9 & $15 \cdot 8$ & 3.7 & $0.183 \dagger$ \\
\hline SFA & & & & & $0.081 \|$ \\
\hline Median & & & & & \\
\hline Range & & & & & \\
\hline Trans-FA & 0.6 & 0.3 & 0.6 & 0.3 & $0.832 \dagger$ \\
\hline$n-6: n-3$ ratio & & & & & $0.017 \|^{* *}$ \\
\hline Median & & & & & \\
\hline Range & & & & & \\
\hline
\end{tabular}


Table 1. Continued

\begin{tabular}{|c|c|c|c|c|c|}
\hline & \multicolumn{2}{|c|}{$\begin{array}{l}\text { Controlled asthma } \\
\qquad(n 40)\end{array}$} & \multicolumn{2}{|c|}{$\begin{array}{l}\text { Non-controlled asthma } \\
(n \text { 134) }\end{array}$} & \multirow[b]{2}{*}{$P$} \\
\hline & Mean & SD & Mean & SD & \\
\hline MUFA:SFA ratio & \multirow{2}{*}{\multicolumn{2}{|c|}{0.86}} & & & $0.325 \|$ \\
\hline Median & & & \multicolumn{2}{|c|}{0.90} & \\
\hline Range & \multicolumn{2}{|c|}{$0.6-1.6$} & \multicolumn{2}{|c|}{$0.5-1.8$} & \\
\hline Ethanol (\% TEV) & \multirow{2}{*}{\multicolumn{2}{|c|}{1.4}} & & & $0.015 \|^{\star *}$ \\
\hline Median & & & \multicolumn{2}{|c|}{3.7} & \\
\hline Range & \multicolumn{2}{|c|}{$0.0-24.8$} & \multicolumn{2}{|c|}{$0.0-37.0$} & \\
\hline Cholesterol (mg) & 644.0 & $209 \cdot 0$ & $590 \cdot 0$ & $245 \cdot 0$ & $0.211 \dagger$ \\
\hline Total dietary fibre (g) & 28.5 & $9 \cdot 2$ & $26 \cdot 0$ & 12.5 & $0.254 \dagger$ \\
\hline Carotene ( $\mu \mathrm{g}$ RAE) & \multirow{2}{*}{\multicolumn{2}{|c|}{$478 \cdot 3$}} & & & $0.360 \|$ \\
\hline Median & & & \multicolumn{2}{|c|}{$430 \cdot 4$} & \\
\hline Range & \multicolumn{2}{|c|}{$113.5-1871.6$} & \multicolumn{2}{|c|}{$52 \cdot 7-2501 \cdot 5$} & \\
\hline Retinol ( $\mu$ g RAE) & 1483.5 & $4384 \cdot 1$ & $1402 \cdot 0$ & $3884 \cdot 0$ & $0.212 \|$ \\
\hline Vitamin $\mathrm{E}(\mathrm{mg} \alpha-\mathrm{TE})$ & $10 \cdot 3$ & 14.4 & 8.5 & $37 \cdot 0$ & $0.074 \|$ \\
\hline Median & \multirow{2}{*}{\multicolumn{2}{|c|}{$\begin{array}{c}10 \cdot 3 \\
5 \cdot 2-19 \cdot 5\end{array}$}} & \multirow{2}{*}{\multicolumn{2}{|c|}{$\begin{array}{c}8.5 \\
2.4-39.4\end{array}$}} & \\
\hline Range & & & & & \\
\hline Vitamin C (mg) & $143 \cdot 7$ & $207 \cdot 8$ & $131 \cdot 0$ & $136 \cdot 1$ & $0.338 \|$ \\
\hline $\mathrm{Mg}(\mathrm{mg})$ & $416 \cdot 9$ & $103 \cdot 4$ & $386 \cdot 3$ & 151.5 & $0.233 \dagger$ \\
\hline $\mathrm{Zn}(\mathrm{mg})$ & $20 \cdot 4$ & $7 \cdot 8$ & $18 \cdot 7$ & $8 \cdot 3$ & $0.259 \dagger$ \\
\hline $\begin{array}{l}\text { MET, metabolic equivalent; IC } \\
\text { volume in the first second; } \\
\text { acid; FA, fatty acid; RAE, re } \\
\text { * Macro- and micronutrients ar } \\
\dagger t \text { test. } \\
\text { ₹ } \chi^{2} \text { test. } \\
\S \text { Sugars refer to all monosac } \\
\text { present in honey, syrups an } \\
\| \text { Mann-Whitney } U \text { test }\end{array}$ & \multicolumn{4}{|c|}{$\begin{array}{l}\ddagger \chi^{2} \text { test. } \\
\S \text { Sugars refer to all monosaccharides and disaccharides added to foods by the manufacturer, cooking or consumer, plus sugars naturally } \\
\text { present in honey, syrups and fruit juices. } \\
\| \text { Mann-Whitney } U \text { test }\end{array}$} & $\begin{array}{l}\text { d expiratory } \\
\text {, } \alpha \text {-linolenic } \\
\text { ars naturally }\end{array}$ \\
\hline
\end{tabular}

\section{Statistical analysis}

Descriptive statistics are expressed as means and standard deviations, and proportions (\%), whereas PA and several nutrient data are presented as medians and ranges given the nonnormal distribution. eNO was logarithmically transformed to attain normal distribution and is presented as geometric means and 95\% CI. Atopic status, defined by positive skin prick tests, medical diagnosis of allergic rhinitis, present use of inhaled corticosteroid, education $(\leq 4,5$ to 9 and $\geq 10$ years) and smoking status (non-smoker, past smoker and present smoker) were also recorded.

Nutritional variables were adjusted for total energy intake using the nutrient residual model ${ }^{(16)}$. In this model, energyadjusted nutrient intakes are computed as the residuals from the regression analysis, with total energy intake as independent variables and absolute intakes as dependent variables. The associations between nutritional intake and asthma outcomes were performed using linear regression, multiple linear regression and unconditional logistic regression models. Linear regression was initially fitted to analyse the associations between nutrient intake (independent variables) and asthma outcomes (dependent variables). Multiple linear regression models adjusted for confounders were performed separately for eNO, FEV1, asthma life quality and ACQ scores (categorical confounder variables were transformed into dummy variables). Logistic regression models were also performed to analyse the associations between nutritional intake and asthma control level. Energy-adjusted nutrients were categorised into tertiles. OR were calculated by reference with the lowest tertile.

Sex, education, age, energy intake, BMI, PA score, smoking, atopy, rhinitis and inhaled corticosteroid were analysed as potential confounders. Only the variables that were significantly associated with each of the asthma outcomes in the univariate analysis were considered in the final regression and logistic models. Considering that smoking status and PA were not significantly associated with eNO, FEV1, asthma life quality or ACQ scores, and that their inclusion as confounders did not influence the effects, these variables were therefore not included in the final models. Considering the biological plausibility related to dietary intake, sex, age and total energy intake, these were considered in all models. A 0.05 level of significance and 95\% CI were considered. Data analysis was performed using the statistical package SPSS $^{\circledR}$, version 17.0 (SPSS Inc., Chicago, IL, USA).

\section{Results}

From the 219 patients invited, forty-five were excluded (twenty-one did not fulfil the inclusion criteria, nine had dietary changes in the last 12 months, eight had incomplete data records, four were considered as energy intake outliers and three refused to participate). Energy intake outliers were previously excluded from the study and were defined as having energy intake values above the arithmetic mean (SD 2), and implausibly low intakes $(<2092 \mathrm{~kJ}(<500 \mathrm{kcal})$ for women and $<3347 \cdot 2 \mathrm{~kJ}(<800 \mathrm{kcal})$ for men). The characteristics of 
Table 2. Associations between nutrient intake and airway inflammation, lung function, asthma quality of life and Asthma Control Questionnaire score (ACQ) ( $\beta$ Coefficients and $95 \%$ confidence intervals)

\begin{tabular}{|c|c|c|c|c|c|c|c|c|}
\hline & \multicolumn{2}{|c|}{ Exhaled NO (ppb) } & \multicolumn{2}{|c|}{ FEV1 (\% predicted) } & \multicolumn{2}{|c|}{ AQL score } & \multicolumn{2}{|c|}{ ACQ score } \\
\hline & \multicolumn{2}{|c|}{ Confounder-adjusted $\dagger$} & \multicolumn{2}{|c|}{ Confounder-adjusted $\ddagger$} & \multicolumn{2}{|c|}{ Confounder-adjusted§ } & \multicolumn{2}{|c|}{ Confounder-adjusted\| } \\
\hline & $\beta$ ๆ & $95 \% \mathrm{Cl}$ & $\beta$ & $95 \% \mathrm{Cl}$ & $\beta$ & $95 \% \mathrm{Cl}$ & $\beta$ & $95 \% \mathrm{Cl}$ \\
\hline \multicolumn{9}{|l|}{ Fatty acids } \\
\hline n-6 PUFA (g) & -0.024 & $-0.035,0.083$ & 0.786 & $-1 \cdot 164,2 \cdot 734$ & 0.035 & $-0.248,0.318$ & -0.037 & $-0.110,0.036$ \\
\hline$n-3$ PUFA (g) & $-0.502^{*}$ & $-0.928,-0.075$ & 9.690 & $-4.373,23.753$ & -1.163 & $-3.222,0.896$ & -0.260 & $-0.792,0.272$ \\
\hline ALA $(g)$ & $-0.357^{*}$ & $-0.608,-0.105$ & 3.180 & $-5.086,11.446$ & -0.998 & $-0.202,0.207$ & -0.283 & $-0.593,0.027$ \\
\hline EPA $(g)$ & -0.003 & $-0.753,0.747$ & 16.056 & $-8.850,40.962$ & -0.422 & $-4.082,3.239$ & -0.232 & $-1.176,0.711$ \\
\hline DHA (g) & -0.005 & $-0.343,0.332$ & 6.477 & $-4 \cdot 752,17 \cdot 706$ & -0.182 & $-1.831,1.467$ & -0.100 & $-0.525,0.325$ \\
\hline $\mathrm{EPA}+\mathrm{DHA}(\mathrm{g})$ & -0.003 & $-0.236,0.230$ & 4.632 & $-3 \cdot 111,12 \cdot 376$ & -0.127 & $-1.265,1.010$ & -0.070 & $-0.363,0.223$ \\
\hline$n-6: n-3$ ratio & 0.053 * & $0.017,0.089$ & -0.072 & $-1 \cdot 278,1 \cdot 134$ & 0.072 & $-0.104,0.248$ & -0.002 & $-0.047,0.044$ \\
\hline MUFA & -0.022 & $-0.050,-0.007$ & -0.222 & $-0.663,1.108$ & 0.028 & $-0.158,0.103$ & -0.007 & $-0.041,0.026$ \\
\hline SFA & $-0.021^{*}$ & $-0.038,-0.004$ & -0.032 & $-0.580,0.515$ & -0.051 & $-0.131,0.029$ & -0.009 & $-0.030,0.011$ \\
\hline MUFA:SFA ratio & $0.637^{*}$ & $0.075,1.199$ & 3.417 & $-14.765,21.600$ & 1.902 & $-0.724,4.529$ & 0.355 & $-0.321,1.030$ \\
\hline \multicolumn{9}{|l|}{ Antioxidant micronutrients } \\
\hline Carotene ( $\mu \mathrm{g}$ RAE) & 0.001 & $-0.000,0.001$ & 0.017 & $-0.009,0.042$ & 0.001 & -0.0050 .003 & -0.001 & $-0.002,0.000$ \\
\hline Retinol ( $\mu$ g RAE) & -0.001 & $-0.001,0.000$ & 0.007 & $-0.006,0.021$ & -0.002 & $-0.004,0.000$ & 0.000 & $-0.001,0.000$ \\
\hline Vitamin $\mathrm{E}$ ( $\mathrm{mg} \alpha-\mathrm{TE})$ & -0.074 & $-0.166,0.019$ & 2.055 & $-0.841,4.950$ & 0.086 & $-0.340,0.519$ & -0.051 & $-0.161,0.058$ \\
\hline Vitamin C (mg) & 0.002 & $-0.002,0.005$ & 0.061 & $-0.056,0.177$ & 0.008 & $-0.009,0.025$ & -0.001 & $-0.006,0.003$ \\
\hline $\mathrm{Mg}(\mathrm{mg})$ & -0.001 & $-0.004,0.003$ & $0 \cdot 106$ & $-0.009,0.222$ & 0.010 & $-0.007,0.027$ & -0.003 & $-0.007,0.002$ \\
\hline $\mathrm{Zn}(\mathrm{mg})$ & 0.006 & $-0.068,0.080$ & 0.159 & $-2.224,2.543$ & -0.095 & $-0.444,0.255$ & -0.008 & $-0.098,0.082$ \\
\hline
\end{tabular}

ppb, Parts per billion; FEV1, forced expiratory volume in the first second; AQL, asthma quality of life; ALA, $\alpha$-linolenic acid.

$\stackrel{P}{P}<0.05$.

† Linear regression; multiple linear regression adjusted for: energy intake, sex, age, BMI, education, rhinitis and atopic status.

‡ Linear regression; multiple linear regression adjusted for: energy intake, sex, age, rhinitis and education.

$\S$ Linear regression; multiple linear regression adjusted for: energy intake, sex, age, BMI, education and inhaled corticosteroid (ICS).

Linear regression; multiple linear regression adjusted for: energy intake, sex, age, education and ICS.

I Representing the adjusted ratio of geometric means. 
Table 3. Association between nutrient intake and asthma control (Odds ratios and $95 \%$ confidence intervals)

\begin{tabular}{|c|c|c|c|c|c|c|}
\hline & \multicolumn{3}{|c|}{ Energy-adjusted $†$} & \multicolumn{3}{|c|}{ Confounder-adjusted $\ddagger$} \\
\hline & OR & $95 \% \mathrm{Cl}$ & $P$ for trend & OR & $95 \% \mathrm{Cl}$ & $P$ for trend \\
\hline \multicolumn{7}{|l|}{ Fatty acids } \\
\hline \multicolumn{7}{|l|}{ n-6 PUFA (g/d) } \\
\hline$<5.19$ & 1.00 & Reference & & 1.00 & Reference & \\
\hline $5 \cdot 19-7 \cdot 12$ & 0.58 & $0.25,1.35$ & & 0.53 & $0.22,1.30$ & \\
\hline$>7.12$ & 1.25 & $0.49,3.18$ & 0.659 & $1 \cdot 23$ & $0.45,3.35$ & 0.707 \\
\hline \multicolumn{7}{|l|}{$n-3$ PUFA (g/d) } \\
\hline$<0.73$ & 1.00 & Reference & & 1.00 & Reference & \\
\hline $0.73-0.94$ & $0.21^{*}$ & $0.07,0.67$ & & $0.18^{*}$ & $0.05,0.62$ & \\
\hline$>0.94$ & $0.14^{\star}$ & $0.04,0.44$ & $0.001^{*}$ & $0.14^{*}$ & $0.04,0.45$ & $0.001^{*}$ \\
\hline \multicolumn{7}{|l|}{$\operatorname{ALA}(g / d)$} \\
\hline$<1.54$ & 1.00 & Reference & & 1.00 & Reference & \\
\hline $1.54-1.96$ & $0.21^{\star}$ & $0.07,0.61$ & & $0.19^{*}$ & $0.06,0.59$ & \\
\hline$>1.96$ & $0.23^{*}$ & $0.08,0.67$ & $0.010^{*}$ & $0 \cdot 18^{*}$ & $0.06,0.58$ & $0.006^{*}$ \\
\hline \multicolumn{7}{|l|}{$\operatorname{EPA}(\mathrm{g} / \mathrm{d})$} \\
\hline$<0.07$ & 1.00 & Reference & & 1.00 & Reference & \\
\hline $0.07-0.14$ & 0.85 & $0.32,2.24$ & & 0.80 & $0.29,2.21$ & \\
\hline$>0.14$ & 0.34 & $0.14,0.83$ & 0.496 & 0.37 & $0.15,0.99$ & 0.689 \\
\hline \multicolumn{7}{|l|}{$\mathrm{DHA}(\mathrm{g} / \mathrm{d})$} \\
\hline$<0.16$ & 1.00 & Reference & & 1.00 & Reference & \\
\hline $0.16-0.30$ & 0.82 & $0.32,2.07$ & & 0.82 & $0.31,2 \cdot 19$ & \\
\hline$>0.30$ & 0.48 & $0.20,1.17$ & 0.086 & 0.55 & $0.22,1.40$ & 0.189 \\
\hline \multicolumn{7}{|c|}{$E P A+D H A(g / d)$} \\
\hline$<0.23$ & 1.00 & Reference & & 1.00 & Reference & \\
\hline $0.23-0.43$ & 0.78 & $0.30,2.02$ & & 0.82 & $0.30,2.21$ & \\
\hline$>0.43$ & 0.21 & $0.18,1.03$ & 0.045 & 0.49 & $0.19,1.23$ & 0.107 \\
\hline \multicolumn{7}{|l|}{$n-6: n-3$ ratio } \\
\hline$<6.45$ & 1.00 & Reference & & 1.00 & Reference & \\
\hline $6 \cdot 45-8 \cdot 11$ & $2 \cdot 18$ & $0.95,4.99$ & & $2 \cdot 24$ & $0.93,5.36$ & \\
\hline$>8 \cdot 11$ & $4 \cdot 14^{\star}$ & $1.59,10.74$ & $0.004^{*}$ & $3.69^{*}$ & $1.37,9.94$ & $0.009^{*}$ \\
\hline \multicolumn{7}{|l|}{ MUFA (g/d) } \\
\hline$<16 \cdot 12$ & 1.00 & Reference & & 1.00 & Reference & \\
\hline $16 \cdot 12-20 \cdot 06$ & 0.58 & $0.23,1.47$ & & 0.50 & $0.19,1.35$ & \\
\hline$>20.06$ & 0.44 & $0.18,1 \cdot 10$ & 0.083 & 0.47 & $0.17,1.27$ & 0.154 \\
\hline \multicolumn{7}{|l|}{$\operatorname{SFA}(g / d)$} \\
\hline$<16.84$ & 1.00 & Reference & & 1.00 & Reference & \\
\hline $16 \cdot 84-23 \cdot 90$ & 0.46 & $0.18,1.19$ & & 0.51 & $0.19,1.34$ & \\
\hline$>23.90$ & $0.39^{\star}$ & $0.15,0.98$ & 0.056 & $0.36^{*}$ & $0.13,0.97$ & $0.047^{\star}$ \\
\hline MUFA:SFA ratic & & & & & & \\
\hline$<0.81$ & 1.00 & Reference & & 1.00 & Reference & \\
\hline $0.81-1.0$ & 1.45 & $0.62,2.39$ & & 1.44 & $0.59,3.52$ & \\
\hline$>1.0$ & 1.69 & $0.71,4.01$ & 0.203 & 1.89 & $0.75,4.78$ & 0.072 \\
\hline Antioxidant micror & & & & & & \\
\hline Carotene $(\mu \mathrm{g} R$ & & & & & & \\
\hline$<152$ & 1.00 & Reference & & 1.00 & Reference & \\
\hline $152-238$ & 1.00 & $0.41,2.46$ & & $1 \cdot 10$ & $0.43,2.77$ & \\
\hline$>238$ & 0.69 & $0.29,1.61$ & 0.310 & 0.76 & $0.31,1.87$ & 0.412 \\
\hline Retinol ( $\mu \mathrm{g} \mathrm{RAE}$ & & & & & & \\
\hline$<95$ & 1.00 & Reference & & 1.00 & Reference & \\
\hline $95-849$ & $0.33^{\star}$ & $0.13,0.83$ & & $0.32^{*}$ & $0.12,0.87$ & \\
\hline$>849$ & 0.55 & $0.21,1.46$ & 0.334 & 0.54 & $0.19,1.50$ & 0.355 \\
\hline Vitamin E (mg o & & & & & & \\
\hline$<3.22$ & 1.00 & Reference & & 1.00 & Reference & \\
\hline $3 \cdot 22-4 \cdot 02$ & 0.89 & $0.35,2.29$ & & 0.84 & $0.31,2.26$ & \\
\hline$>4.02$ & 0.43 & $0.18,1.03$ & $0.049^{*}$ & 0.43 & $0.17,1.10$ & 0.079 \\
\hline Vitamin C $(\mathrm{mg} / \mathrm{c}$ & & & & & & \\
\hline$<52$ & 1.00 & Reference & & 1.00 & Reference & \\
\hline $52-66$ & 0.83 & $0.35,1.94$ & & 0.84 & $0.35,2.04$ & \\
\hline$>66$ & $1 \cdot 11$ & $0.46,2.69$ & 0.740 & $1 \cdot 17$ & $0.46,3.02$ & 0.667 \\
\hline$M g(m g / d)$ & & & & & & \\
\hline$<149$ & 1.00 & Reference & & 1.00 & Reference & \\
\hline $149-175$ & 0.66 & $0.26,1.63$ & & 0.72 & $0.28,1.85$ & \\
\hline$>175$ & 0.55 & $0.22,1.33$ & 0.184 & 0.63 & $0.25,1.61$ & 0.338 \\
\hline $\mathrm{Zn}(\mathrm{mg} / \mathrm{d})$ & & & & & & \\
\hline$<5.73$ & 1.00 & Reference & & 1.00 & Reference & \\
\hline $5 \cdot 73-7 \cdot 26$ & 0.84 & $0.35,2.01$ & & 0.97 & $0.38,2.45$ & \\
\hline$>7.26$ & 0.80 & $0.33,1.92$ & 0.606 & 0.79 & $0.31,2.01$ & 0.657 \\
\hline
\end{tabular}

ALA, $\alpha$-linolenic acid, RAE, retinol A equivalents.

${ }^{\star} P<0.05$.

$\dagger$ Logistic regression adjusted for energy intake.

‡ Logistic regression adjusted for energy intake, sex, age, education and inhaled corticosteroid. 
excluded patients, regarding age, education, smoking status and asthma severity, were similar to the 174 patients (81\%) included in the analysis.

According to asthma control definition, 23 and $77 \%$ of the subjects were classified, respectively, as having controlled and non-controlled asthma (Table 1). Considering the energy contribution of macronutrients, no significant differences between these two groups were observed for total carbohydrates, total fat and SFA intake. However, controlled patients had a significantly lower $n-6: n-3$ PUFA ratio intake compared with non-controlled patients $(P=0 \cdot 017)$ and had a significantly higher intake of ALA $(P=0 \cdot 022)$, EPA $(P=0 \cdot 016)$, DHA $(P=0.021)$ and EPA + DHA $(P=0.020)$; dietary intakes of $n$-3 PUFA $(P=0.090)$, SFA $(P=0.081)$ and vitamin $\mathrm{E}$ $(P=0.074)$ were higher in controlled asthmatics, but these differences were not statistically significant.

The associations of the dietary intake of fatty acids and antioxidant micronutrients with markers of asthma adjusted for energy intake, sex, age, BMI, education, atopy, rhinitis and inhaled corticosteroids are presented in Table 2. Higher $n-6: n-3$ PUFA and MUFA:SFA ratios were associated with higher eNO, whereas higher intakes of $n-3$ PUFA and SFA were associated with lower eNO. Higher ALA intake was associated with lower eNO, even after adjustment for marine $n-3$ PUFA ( $R-0.356$, 95\% CI $-0.609,-0 \cdot 105 ; P=0.006)$. No significant associations were found for EPA + DHA and asthma outcomes. Energy-adjusted MUFA and Mg were associated with eNO and FEV1, respectively; however, after adjusting for confounders, these associations were no longer significant.

The OR for asthma control accordingly with the dietary intake of fatty acids and antioxidant micronutrients are given in Table 3. Intake of $n$-3 PUFA between 0.73 and $0.94 \mathrm{~g} / \mathrm{d}$ and above $0.94 \mathrm{~g} / \mathrm{d}$ reduced the odds of non-controlled asthma (second tertile: OR $0 \cdot 18,95 \%$ CI 0.05, 0.62; third tertile: OR 0.14; $95 \%$ CI 0.04, 0.45; $P$ for trend $=0 \cdot 001$ ), while $n-6: n-3$ PUFA above 8.45 had the opposite effect (third tertile: OR 3.69, 95\% CI 1.37, 9.94; $P$ for trend=0.009), after adjusting for energy intake, sex, age, education and inhaled corticosteroids. Dietary intake of ALA between 1.54 and 1.96 and above $1.96 \mathrm{~g} / \mathrm{d}$ reduced the odds of non-controlled asthma (second tertile: OR $0 \cdot 19,95 \%$ CI $0.06,0.59$; third tertile: OR 0.18, 95\% CI 0.06 , 0.58 ; $P$ for trend=0.006), after adjusting for confounders. After adjusting also for alternate $n$ - 3 PUFA, the protective effect of ALA in asthma control still remained significant (second tertile: OR $0 \cdot 19,95 \%$ CI $0 \cdot 06,0 \cdot 60$; third tertile: OR $0 \cdot 18,95 \%$ CI $0.06,0.58 ; P$ for trend $=0.005$ ), independent of $\mathrm{EPA}+\mathrm{DHA}$. No significant association was observed for $\mathrm{EPA}+$ DHA and asthma control.

The higher intake of SFA $(>23.9 \mathrm{~g} / \mathrm{d})$ also decreased the probability of having non-controlled asthma (OR 0.36, 95\% CI $0 \cdot 13,0 \cdot 97 ; P$ for trend $=0 \cdot 047$ ), after controlling for confounders. Considering micronutrients, dietary intake of retinol between 95 and $849 \mu \mathrm{g}$ retinol A equivalents/d decreased the odds of having non-controlled asthma; however, the trend according to the retinol intake category was not significant ( $P$ for trend $=0 \cdot 355$ ). A protective trend was also observed for energy-adjusted vitamin $\mathrm{E}$ and asthma control (OR 0.43,
95\% CI $0 \cdot 18,1.03 ; P=0.049)$; however, this association was no longer significant after the final adjustment.

\section{Discussion}

In the present study, higher dietary intakes of $n-3$ PUFA and SFA were associated with a decreased levels of eNO and improved likelihood of asthma being under control, while a high ratio of $n-6: n-3$ PUFA had the opposite effect. In addition, higher dietary intake of ALA was associated with lower eNO and reduced the likelihood of non-controlled asthma, independent of marine $n$ - 3 PUFA. No significant associations between the dietary intake of EPA + DHA and antioxidant vitamins and minerals and asthma outcomes were observed.

The present results are limited by the cross-sectional design of the study which leaves open any possible cause-effect relationship and the role of other factors. Nevertheless, an inverse causal relationship is not probable and we assessed established lifestyle factors that could have an important role in asthma and that influence nutrient intake, such as total energy intake, PA and BMI, and the association between nutrients and asthma outcomes was extensively adjusted for confounders.

To the best of our knowledge, this is the first study exploring the association between different types of dietary fatty acids and antioxidant nutrient intake, and asthma control. Moreover, we assess the dietary intake of vegetable (ALA) and marine (EPA + DHA) $n-3$ PUFA, and report for the first time the protective effect of ALA in asthma control. The score we used to assess control, which included different dimensions of the disease such as inflammation, lung function and symptoms, has been shown to explain $77 \%$ of the variability of asthma control ${ }^{(5)}$. Another important strength of the present study was the FFQ that we used, since it has been validated for Portuguese adults ${ }^{(6)}$, and it has been shown to provide reliable estimates for $n-3$ PUFA and SFA ${ }^{(6)}$.

In the present study, a higher dietary intake of $n$ - 3 PUFA $(>0.94 \mathrm{~g} / \mathrm{d})$ and ALA $(>1.96 \mathrm{~g} / \mathrm{d})$ reduced the odds of noncontrolled asthma. Considering that the prevalence of noncontrolled asthma in the present study was high, the OR may be biased towards overestimating the risk. Nevertheless, even though we could admit an overestimation of the protective effect, the reverse result should not be expected.

ALA is the major plant-based $n-3$ PUFA and exerts main effects through conversion to EPA and DHA, when dietary intake of marine PUFA is low ${ }^{(17,18)}$. Long-chain $n-3$ PUFA decreases the production of inflammatory mediators, competitively inhibiting the metabolism of arachidonic acid (generating less active prostenoids and leukotrienes), suppressing IgE production, and thereby potentially acting to reduce airway inflammation and bronchoconstriction in asthma ${ }^{(18,19)}$. However, results were inconclusive. A systematic review from Cochrane of the clinical effects of $n$-3 PUFA fish oil supplementation in established asthma suggests that the results are not consistent and that there is little evidence to recommend such supplementation in order to improve asthma control ${ }^{(20)}$. Reconciling the data from experimental and observational studies is difficult, most probably, due to 
different methods of assessment of dietary intake and different definitions of asthma. Taken the data into account from previous cross-sectional studies, it seems that dietary or serum n-3 PUFA levels are directly associated with lung function, at least in asthmatics ${ }^{(21-23)}$ and atopy ${ }^{(24)}$, and are protective for the risk of asthma or atopy. Recently, in a large populationbased study, asthma risk was doubled in subjects who had never eaten fish during childhood and a minimum of weekly fish intake in adulthood was protective against asthma symptoms ${ }^{(25)}$. In a small study, fish oil supplementation failed to provide any benefit in eNO, lung function or asthma control in asthmatic women ${ }^{(26)}$. In the present study, dietary intake of $n-3$ PUFA and ALA was associated with improved asthma control and lower eNO, independent of EPA + DHA. Higher intake of ALA (and also not EPA or DHA) was previously associated with a decreased risk of allergic sensitisation and allergic rhinitis in adults ${ }^{(27)}$. However, the link between ALA and asthma is still poorly addressed. In the present study, dietary intake of EPA and DHA is very similar between controlled and non-controlled subjects, and we have found no significant associations between EPA or DHA and asthma outcomes. There is evidence suggesting that ALA, EPA and DHA might have heterogeneous and potentially independent effects on inflammation, gene expression and chronic diseases; therefore, a better understanding of the individual role of $n-3$ PUFA in inflammatory diseases, such as asthma, is needed ${ }^{(17,18)}$. It has been suggested that higher margarine intake rich in $n-6$ PUFA is associated with an increased risk of asthma ${ }^{(28,29)}$ and hay fever ${ }^{(30)}$ in adulthood, and eczema and allergic sensitisation in children ${ }^{(31)}$. Dietary intake of $n$ - 6 PUFA was similar among controlled and noncontrolled subjects, and therefore, no significant associations for $n-6$ PUFA and asthma outcomes were observed. Nevertheless, the ratio of $n-6: n-3$ PUFA above $8 \cdot 45$, which was more than tripled the odds of non-controlled asthma, was associated with increased levels of eNO.

In the present study, we analysed the total SFA intake, irrespective of the specific types. However, different types of SFA could have different effects. Foods high in SFA, such as butter $^{(32)}$, whole milk ${ }^{(32,33)}$ and non-pasteurised farm milk ${ }^{(34-36)}$, have been consistently associated with a reduced risk of asthma. For milk, it is not clear whether associations should be attributed to SFA, vitamin A or even to microbial agents (in the case of whole non-pasteurised milk or farmrelated co-exposures $)^{(34-36)}$. Therefore, the present results on SFA could also be a proxy of a dietary pattern high in milk and dairy products. Several epidemiological studies have reported beneficial associations for higher intake of nutrients that may be relevant in the redox mechanisms, such as vitamin $\mathrm{C}^{(21,37,38)}$, vitamin $\mathrm{E}^{(37,39)}$, carotenoids ${ }^{(40,41)}$, $\mathrm{Se}^{(7)}$ and $\mathrm{Mg}^{(42)}$. However, these findings are not conclusive ${ }^{(43)}$ and intervention studies with single nutrient supplementation have been disappointing ${ }^{(44-47)}$. Inverse associations with asthma have also been observed for foods rich in these micronutrients, such as fresh fruit ${ }^{(7,33,48,49)}$ and vegetables $^{(48)}$, and nuts ${ }^{(4,50,51)}$, and additional benefits may arise from the synergistic effects between nutrients in foods and specific dietary patterns. Nuts contain a high proportion of ALA, fibre, vitamins, minerals and many bioactive compounds that may modulate redox status, and inflammatory and immune responses ${ }^{(30,52)}$. We have previously reported that intake of nuts is positively associated with lung function, and high adherence to an overall healthy dietary pattern, such as the Mediterranean diet, is associated with an improved asthma control in adults, independent of other lifestyle factors $^{(4)}$.

In summary, the present results provide additional support for the benefits of adequate dietary advice and give a rationale to nutritional intervention studies in asthmatics. Healthy eating in asthma, providing foods high in ALA, such as nuts, and an adequate balance between $n-6$ and $n$-3 PUFA, may reduce disease severity and improve asthma control, independent of other lifestyle factors ${ }^{(4)}$.

\section{Acknowledgements}

We thank all patients who participated in the present study; to Sandra Gomes for assistance with patient recruitment and to Milton Severo for support with statistical analyses. R. B., A. M., J. F. and M. G. C.-B. were responsible for patient enrolment and data collection; R. B. and A. M. conducted the data analyses; J. F., C. L. and P. M. advised on the statistical analysis; C. L. and P. M. advised on the nutritional analyses and supervised the study; R. B., A. M., L. D., T. H., C. L. and P. M. interpreted and critically discussed the results; R. B. wrote the first draft and all authors contributed to and critically reviewed the manuscript. The present study received no specific grant from any funding agency in the public, commercial or not-for-profit sectors. None of the authors has any conflicts of interest.

\section{References}

1. Devereux G (2006) The increase in the prevalence of asthma and allergy: food for thought. Nat Rev Immunol 6, 869-874.

2. Black PN \& Sharpe S (1997) Dietary fat and asthma: is there a connection? Eur Respir J 10, 6-12.

3. Seaton A, Godden DJ \& Brown K (1994) Increase in asthma - a more toxic environment or a more susceptible population. Thorax 49, 171-174.

4. Barros R, Moreira A, Fonseca J, et al. (2008) Adherence to the Mediterranean diet and fresh fruit intake are associated with improved asthma control. Allergy 63, 917-923.

5. Lopes C, Fonseca J, Delgado L, et al. (2008) Assessing asthma control: questionnaires and exhaled nitric oxide provide complementary information. Eur Respir J 32, 1419-1420.

6. Lopes C, Aro A, Azevedo A, et al. (2007) Intake and adipose tissue composition of fatty acids and risk of myocardial infarction in a male Portuguese community sample. $J \mathrm{Am}$ Diet Assoc 107, 276-286.

7. Shaheen SO, Sterne JA, Thompson RL, et al. (2001) Dietary antioxidants and asthma in adults: population-based casecontrol study. Am J Respir Crit Care Med, 164, 1823-1828.

8. Craig CL, Marshall AL, Sjostrom M, et al. (2003) International physical activity questionnaire: 12 -country reliability and validity. Med Sci Sports Exerc 35, 1381-1395.

9. GINA Workshop Report (2006) Global strategy for asthma management and prevention. http://www.ginasthma.org/ 
Guidelineitem.asp??11=2\&12=1\&intId=60 (cited 20 July 2007).

10. Smith AD, Cowan JO, Brassett KP, et al. (2005) Use of exhaled nitric oxide measurements to guide treatment in chronic asthma. N Engl J Med 352, 2163-2173.

11. Juniper EF, Bousquet J, Abetz L, et al. (2006) Identifying 'well-controlled' and 'not well-controlled' asthma using the Asthma Control Questionnaire. Respir Med 100, 616-621.

12. Fonseca JA, Costa-Pereira A, Delgado L, et al. (2005) Pulmonary function electronic monitoring devices: a randomized agreement study. Chest 128, 1258-1265.

13. American Thoracic Society (1999) Recommendations for standardized procedures for the on-line and off-line measurement of exhaled lower respiratory nitric oxide and nasal nitric oxide in adults and children - 1999. Am J Respir Crit Care Med 160, 2104-2117.

14. Juniper EF, O’Byrne PM, Guyatt GH, et al. (1999) Development and validation of the questionnaire to measure asthma control. Eur Resp J 14, 902-907.

15. Fonseca JA, Delgado L, Costa-Pereira A, et al. (2004) Evaluation of the asthma life quality test for the screening and severity assessment of asthma. Allergy 59, 1198-1204.

16. Willett WC, Howe GR \& Kushi LH (1997) Adjustment for total energy intake in epidemiologic studies. Am J Clin Nutr $\mathbf{6 5}$, Suppl. 4, 1220S-1228S.

17. Anderson BM \& Ma DW (2009) Are all $n$-3 polyunsaturated fatty acids created equal? Lipids Health Dis $\mathbf{8}, 33$.

18. Galli C \& Calder PC (2009) Effects of fat and fatty acid intake on inflammatory and immune responses: a critical review. Ann Nutr Metab 55, 123-139.

19. Calder PC (2006) n-3 Polyunsaturated fatty acids, inflammation, and inflammatory diseases. Am J Clin Nutr 83, Suppl. 6, 1505S-1519S.

20. Thien FCK, De Luca S, Woods R, et al. (2002) Dietary marine fatty acids (fish oil) for asthma in adults and children. In Cochrane Database of Systematic Reviews, issue 2, CD001283.

21. de Luis DA, Armentia A, Aller R, et al. (2005) Dietary intake in patients with asthma: a case control study. Nutrition $\mathbf{2 1}$, 320-324.

22. Kompauer I, Demmelmair H, Koletzko B, et al. (2008) Association of fatty acids in serum phospholipids with lung function and bronchial hyperresponsiveness in adults. Eur J Epidemiol 23, 175-190.

23. Tabak C, Wijga AH, de Meer G, et al. (2006) Diet and asthma in Dutch school children (ISAAC-2). Thorax 61, 1048-1053.

24. Chatzi L, Torrent M, Romieu I, et al. (2007) Diet, wheeze, and atopy in school children in Menorca, Spain. Pediatr Allergy Immunol 18, 480-485.

25. Laerum BN, Wentzel-Larsen T, Gulsvik A, et al. (2007) Relationship of fish and cod oil intake with adult asthma. Clin Exp Allergy 37, 1616-1623.

26. Moreira A, Moreira P, Delgado L, et al. (2007) Pilot study of the effects of $n-3$ polyunsaturated fatty acids on exhaled nitric oxide in patients with stable asthma. J Investig Allergol Clin Immunol 17, 309-313.

27. Hoff S, Seiler H, Heinrich J, et al. (2005) Allergic sensitisation and allergic rhinitis are associated with $n-3$ polyunsaturated fatty acids in the diet and in red blood cell membranes. Eur J Clin Nutr 59, 1071-1080.

28. Nagel G \& Linseisen J (2004) Dietary intake of fatty acids, antioxidants and selected food groups and asthma in adults. Eur J Clin Nutr 59, 8-15.

29. Bolte G, Winkler G, Holscher B, et al. (2005) Margarine consumption, asthma, and allergy in young adults: results of the
German National Health Survey 1998. Ann Epidemiol 15, 207-213.

30. Trak-Fellermeier MA, Brasche S, Winkler G, et al. (2004) Food and fatty acid intake and atopic disease in adults. Eur Respir J 23, 575-582.

31. Sausenthaler S, Kompauer I, Borte M, et al. (2006) Margarine and butter consumption, eczema and allergic sensitization in children. The LISA birth cohort study. Pediatr Allergy Immunol 17, 85-93.

32. Wijga AH, Smit HA, Kerkhof M, et al. (2003) Association of consumption of products containing milk fat with reduced asthma risk in pre-school children: the PIAMA birth cohort study. Thorax 58, 567-572.

33. Woods RK, Walters EH, Raven JM, et al. (2003) Food and nutrient intakes and asthma risk in young adults. Am J Clin Nutr 78, 414-421.

34. Waser M, Michels KB, Bieli C, et al. (2007) Inverse association of farm milk consumption with asthma and allergy in rural and suburban populations across Europe. Clin Exp Allergy 37, 661-670.

35. Riedler J, Braun-Fahrlander C, Eder W, et al. (2001) Exposure to farming in early life and development of asthma and allergy: a cross-sectional survey. Lancet 358, 1129-1133.

36. Perkin MR \& Strachan DP (2006) Which aspects of the farming lifestyle explain the inverse association with childhood allergy? J Allergy Clin Immunol 117, 1374-1381.

37. Bodner C, Godden D, Brown K, et al. (1999) Antioxidant intake and adult-onset wheeze: a case-control study. Aberdeen WHEASE Study Group. Eur Respir J 13, 22-30.

38. Patel BD, Welch AA, Bingham SA, et al. (2006) Dietary antioxidants and asthma in adults. Thorax 61, 388-393.

39. Troisi RJ, Willett WC, Weiss ST, et al. (1995) A prospective study of diet and adult-onset asthma. Am J Respir Crit Care Med 151, 1401-1408.

40. Ochs-Balcom HM, Grant BJB, Muti P, et al. (2006) Antioxidants, oxidative stress, and pulmonary function in individuals diagnosed with asthma or COPD. Eur J Clin Nutr 60, 991-999

41. Schunemann HJ, McCann S, Grant BJB, et al. (2002) Lung function in relation to intake of carotenoids and other antioxidant vitamins in a population-based study. Am J Epidemiol 155, 463-471.

42. Britton J, Pavord I, Richards K, et al. (1994) Dietary magnesium, lung-function, wheezing, and airway hyperreactivity in a random adult-population sample. Lancet $\mathbf{3 4 4}$, 357-362.

43. Allen S, Britton JR \& Leonardi-Bee JA (2009) Association between antioxidant vitamins and asthma outcome measures: systematic review and meta-analysis. Thorax $\mathbf{6 4}$, 610-619.

44. Dunstan JA, Breckler L, Hale J, et al. (2007) Supplementation with vitamins C, E, beta-carotene and selenium has no effect on anti-oxidant status and immune responses in allergic adults: a randomized controlled trial. Clin Exp Allergy 37, $180-187$

45. Fogarty A, Lewis SA, Scrivener SL, et al. (2003) Oral magnesium and vitamin $C$ supplements in asthma: a parallel group randomized placebo-controlled trial. Clin Exp Allergy 33, 1355-1359.

46. Ram FSF, Rowe BH \& Kaur B (2004) Vitamin C supplementation for asthma. In Cochrane Database of Systematic Reviews, issue 3, CD000993.

47. Pearson PJK, Lewis SA, Britton J, et al. (2004) Vitamin E supplements in asthma: a parallel group randomised placebo controlled trial. Thorax 59, 652-656. 
48. Romieu I, Varraso R, Avenel V, et al. (2006) Fruit and vegetable intakes and asthma in the E3N study. Thorax 61, 209-215.

49. Patel BD, Welch AA, Bingham SA, et al. (2006) Dietary antioxidants and asthma in adults. Thorax 61, 388-393.

50. Chatzi L, Apostolaki G, Bibakis I, et al. (2007) Protective effect of fruits, vegetables and the Mediterranean diet on asthma and allergies among children in Crete. Thorax 62 , $677-683$
51. Garcia-Marcos L, Canflanca IM, Garrido JB, et al. (2007) Relationship of asthma and rhinoconjunctivitis with obesity, exercise and Mediterranean diet in Spanish schoolchildren. Thorax 62, 503-508.

52. Kris-Etherton PM, Yu-Poth S, Sabate J, et al. (1999) Nuts and their bioactive constituents: effects on serum lipids and other factors that affect disease risk. Am J Clin Nutr 70, Suppl. 3, 504S-511S 